\title{
EXTENDED PENALTY COEFFICIENTS FOR ELIMINATION THE LOCKING EFFECTS IN MODERATELY THICK BEAM AND PLATE FINITE ELEMENTS
}

\author{
W. GILEWSKI ${ }^{1}$
}

\begin{abstract}
The present paper is dedicated to presentation and energy verification of the methods of stabilization the strain energy by penalty coefficients. Verification of the methods is based on the consistency and ellipticity conditions to be satisfied by the finite elements. Three methods of stabilization are discussed. The first does not satisfy the above requirements. The second is consistent but cannot eliminate parasitic energy terms. The third method, proposed by the author, is based on the decomposition of the element stiffness matrix. The method can help to eliminate locking of the finite elements. For two-noded beam element with linear shape functions and exact integration a stabilized free of locking (and elliptical) element is received (equivalent to reduced integration element). Two plate finite elements are analyzed: four-noded rectangular element and DSG triangle. A new method of stabilization with the use of four independent parameters is proposed. The finite elements with this kind of stabilization satisfy the consistency condition. In the rectangular element it was not possible to eliminate one parasitic term of energy which appears during the procedure. For DSG triangle all parasitic terms of energy are eliminated. The penalty coefficients depends on the geometry of the triangle.
\end{abstract}

Keywords: finite elements, shear locking, stabilization, consistency

\section{INTRODUCTION}

Design of finite elements for shells, plates and beams of moderate thickness is one of the most demanding area in the finite element method for many years. In the existing finite elements one can frequently observe the phenomena of locking and parasitic strains. There are a lot of methods for design of finite elements free from locking. An extensive bibliography is collected and discussed in the reference Gilewski [8] and other publications (i.e. Dhanajaya et al. [5], Rezaiee-Pajand et al. [14]). Among others, the method of stabilization of the finite element strain energy by selection of multipliers is an interesting idea (Bischoff and Bletzinger [1,2], Bletzinger, Bischoff and Ramm [3], Carpenter, Belytschko and Stolarski [4], Fried [6], Lyly, Stenberg and Vihinen [12], Mohr [13], Tessler [15]). Correct finite element should satisfy the consistency, ellipticity

1 Faculty of Civil Engineering, Warsaw University of Technology, Al. Armii Ludowej 16, 00-637 Warsaw, Poland, e-mail: w.gilewski@il.pw.edu.pl 
in inf-sup conditions for mixed as well as displacement based FE models (Iosilevich et al. [11], Gilewski [8]).

Consistency condition means that the quadratic form of the strain energy in the finite element formulation, which depends on the typical element dimension $a$, should be equivalent to the bi-linear form of the mathematical model in the limit case $a \rightarrow 0$. Ellipticity condition is the main condition for existence and synonymous of the FE solution. This condition is related to the strain energy properties and can be checked by the analysis of eigenvalues and eigenvectors of a single unsupported element. Inf-sup condition is difficult to be analyzed analytically, can be checked numerically (Gilewski, Sitek [10]) and will not be considered below.

The objective of the present paper is to check the correctness of the use the strain energy multipliers to eliminate shear locking, which is commonly used in commercial finite element systems. The criterion of the correctness is satisfying the consistency condition. The ellipticity condition can be checked in the second phase of the analysis. In the $1^{\text {st }}$ part of the paper the coefficients existing in the literature are evaluated. The new, original way for construction the multipliers are presented and evaluated in the $2^{\text {nd }}$ part. A couple of well known finite elements for Timoshenko beam and Mindlin plate are analyzed and modified.

\section{FEM ENERGY MULTIPLIERS}

One of the method of elimination (stabilization) of shear locking (or parasitic shear) for moderately thick beams and plates is the use of the strain energy multipliers (see i.e. Bischoff and Bletzinger [1,2], Bletzinger, Bischoff and Ramm [3], Carpenter, Belytschko and Stolarski [4], Fried [6], Lyly, Stenberg and Vihinen [12], Mohr [13], Tessler [15]). Let us consider how it works. The stiffness matrix of beam or plate finite element can be expressed as a sum

$$
\mathbf{K}=\mathbf{K}_{b}+\mathbf{K}_{s},
$$

where $\mathbf{K}_{b}$ is a part of stiffness matrix related to bending and $\mathbf{K}_{s}$ is related to shear. The method of the multipliers of strain energy, known in the literature of the subject, is the following modification of the finite element stiffness matrix

$$
\mathbf{K}=\mathbf{K}_{b}+\alpha \mathbf{K}_{s} .
$$

The coefficient $\alpha$ should be sensitive for geometrical parameters of the element and selected to minimize the effect of locking. Let us consider the geometric parameter for Timoshenko beam elements in the form of a proportion of the bending rigidity $E J$ to shear rigidity $H, \gamma=\frac{E J}{H a^{2}}$, where $a$ is half of the length of the element. This parameter 
is depending on $\left(\frac{h}{a}\right)^{2}$, where $h$ is the beam thickness, and is slightly sensitive for the shape of cross section. The easiest way of construction the coefficient $\alpha$ (see Bischoff and Bletzinger [1], Bletzinger, Bischoff and Ramm [3], Gilewski [8,9]) is the following

$$
\alpha=\alpha\left(\frac{h}{a}\right)=c \gamma,
$$

where $c$ is especially selected constant. Using this coefficient one can control the share of the transverse shear strain energy in the finite element solution. The value of the constant $c$, is usually defined after extensive numerical analysis.

The other method for selection of the coefficient $\alpha$ was proposed by Lyly, Stenberg and Vihinen [12], and extended by Bishoff and Bletzinger [1,2]:

$$
\alpha=\alpha\left(\frac{h}{a}\right)=\frac{1}{1+c / \gamma} .
$$

This coefficient does not disturb the strain energy when the dimension of the element tends to be zero. It is well seen when we expand the coefficient into Taylor power series with respect to the parameter $\frac{c}{\gamma}$

$$
\alpha=\alpha\left(\frac{c}{\gamma}\right)=\frac{1}{1+c / \gamma}=1-\frac{c}{\gamma}+\ldots=1-c \frac{H a^{2}}{E J}+\ldots
$$

The suitable selection of the constant $c$ allow to reduce the influence of transverse shear strain energy. It is recommended to take $c=0.1$ (Bischoff and Bletzinger [1]).

The energy criterion of the correctness of the FEM formulation (described in details and used for evaluation of many finite elements in papers (Gilewski [7-9])) allow to check the strain energy terms for which the coefficient $c$ has the influence and to verify if the correctly selected coefficient can help to cancel the element locking or parasitic shear. In the next part of the paper one can propose the extension of the ideas presented in (Gilewski [8], Bischoff and Bletzinger [2]) and for the following form of stabilized finite element stiffness matrices

$$
\mathbf{K}=\beta \mathbf{K}_{b}+\alpha \mathbf{K}_{s},
$$

where

$$
\beta=1-\frac{d}{\gamma}+\ldots, \quad \alpha=1-\frac{c}{\gamma}+\ldots
$$


Numerical constants $d$ and $c$ should be duly selected to cancel the element dysfunctions. The procedure for development of the stabilized stiffness matrices is the following:

Step 1 .

Define the element stiffness matrix in the form of Eq. (2.1).

Step 2.

Verify the parasitic terms of the strain energy with the use of stabilized matrices in Eqs. (2.6)-(2.7).

Step 3.

Select the constants $d$ and $c$ to cancel the parasitic strain energy terms.

The procedure presented above can be used to select the best stabilized coefficients without necessity of calculating time consuming examples. The examples can only confirm that the constants were selected correctly and locking is canceled.

\section{Selected Timoshenko beam and Mindlin plate finite elements}

The subject under consideration is moderately thick Timoshenko beam with the thickness $h$, bending rigidity $E J$, shear rigidity $H=k G A$ ( $E$ - Young modulus, $G$ - Kirchhoff modulus, $A$ - area of the cross-section, $J$ - moment of inertia of the cross-section, $k$ - shear correction factor). In the theory we have displacement, strain and internal forces defined as:

$$
\begin{aligned}
\mathbf{u}(x) & =\{w(x), \phi(x)\}, \\
\boldsymbol{\varepsilon}(x) & =\{\kappa(x), \chi(x)\}, \\
\boldsymbol{\sigma}(x) & =\{M(x), T(x)\},
\end{aligned}
$$

where: $w$ - transverse displacement, $\phi$ - average angle of the cross section, $\kappa$ - curvature, $\chi$ - transverse shear strain, $M$ - bending moment, $T$ - shear force.

Basic equations of Timoshenko beam in the matrix form are the following

$$
\boldsymbol{\varepsilon}=\mathbf{D u}, \quad \boldsymbol{\sigma}=\mathbf{E} \boldsymbol{\varepsilon}, \quad \mathbf{D}=\left[\begin{array}{cc}
0 & \frac{d}{d x} \\
\frac{d}{d x} & -1
\end{array}\right], \quad \mathbf{E}=\left[\begin{array}{cc}
E J & 0 \\
0 & H
\end{array}\right] .
$$

Strain energy of the beam can be expressed as

$$
E_{s}=\int_{x}\left[E J\left(\frac{d \phi}{d x}\right)^{2}+H\left(\frac{d w}{d x}-\phi\right)^{2}\right] d x
$$


The subject under consideration is also moderately thick Mindlin plate with constant thickness $h$, in the rectangular co-ordinate system $x, y, z$. In the present theory we have displacement, strain and internal forces in the following form:

$$
\begin{aligned}
& \mathbf{u}(x, y)=\left\{w, \phi_{x}, \phi_{y}\right\}, \\
& \boldsymbol{\varepsilon}(x, y)=\left\{\boldsymbol{\kappa}_{x}, \boldsymbol{\kappa}_{y}, \boldsymbol{\kappa}_{x y}, \gamma_{x}, \gamma_{y}\right\}, \\
& \boldsymbol{\sigma}(x, y)=\left\{M_{x}, M_{y}, M_{x y}, T_{x}, T_{y}\right\},
\end{aligned}
$$

where: $w$ - transverse displacement, $\phi_{x}, \phi_{y}$ - average fiber angles, $\kappa_{x}, \kappa_{y}, \kappa_{x y}$ curvatures, $\gamma_{x}, \gamma_{y}$ - transverse shear strains, $M_{x}, M_{y}, M_{x y}$ - bending and twisting moments, $T_{x}, T_{y}$ - shear forces, $D=\frac{E h^{3}}{12\left(1-v^{2}\right)}, H=\frac{k E h}{2(1+v)}, E-$ Young modulus, $v-$ Poisson ratio, $k-$ transverse shear strain correction factor.

Basic equations of Mindlin plate in the matrix form are the following

$$
\mathbf{D}=\left[\begin{array}{ccc}
0 & \frac{\partial}{\partial x} & 0 \\
0 & 0 & \frac{\partial}{\partial y} \\
0 & \frac{\partial}{\partial y} & \frac{\partial}{\partial x} \\
\frac{\partial}{\partial x} & -1 & 0 \\
\frac{\partial}{\partial y} & 0 & -1
\end{array}\right], \quad \mathbf{E}=\left[\begin{array}{ccccc}
D & v D & 0 & 0 & 0 \\
v D & D & 0 & 0 & 0 \\
0 & 0 & \frac{1-v}{2} D & 0 & 0 \\
0 & 0 & 0 & H & 0 \\
0 & 0 & 0 & 0 & H
\end{array}\right] .
$$

The strain energy of the plate should be expressed in the form

$$
E_{s}=\frac{1}{2} \int_{\Omega}\left\{\begin{array}{l}
D\left(\frac{\partial \phi_{x}}{\partial x}\right)^{2}+D \frac{1-v}{2}\left(\frac{\partial \phi_{x}}{\partial y}\right)^{2}+D\left(\frac{\partial \phi_{y}}{\partial y}\right)^{2}+D \frac{1-v}{2}\left(\frac{\partial \phi_{y}}{\partial x}\right)^{2}+ \\
+2 v D \frac{\partial \phi_{x}}{\partial x} \frac{\partial \phi_{y}}{\partial y}+D(1-v) \frac{\partial \phi_{y}}{\partial x} \frac{\partial \phi_{x}}{\partial y}+H\left[\left(\phi_{x}-\frac{\partial w}{\partial x}\right)^{2}+\left(\phi_{y}-\frac{\partial w}{\partial y}\right)^{2}\right]
\end{array}\right\} d \Omega .
$$

In the present paper one beam and two plate finite elements are considered. 
3.1. 2-NODED ELEMENT BEAM ELEMENT WITH LINEAR SHAPE FUNCTIONS AND EXACT INTEGRATION

Let us consider Timoshenko beam finite element which demonstrates parasitic strain energy (see Fried [6]). Stiffness matrix of the 2-node beam element of the length $2 a$, natural d.o.f. $\mathbf{q}=\left\{w_{1}, \phi_{1}, w_{2}, \phi_{2}\right\}$, linear shape functions and exact integration can be expressed as

$$
\mathbf{K}^{2 W-F U L L}=\frac{H}{2 a}\left[\begin{array}{cccc}
1 & a & -1 & a \\
a & a^{2}\left(\frac{4}{3}+\gamma\right) & -a & a^{2}\left(\frac{2}{3}-\gamma\right) \\
-1 & -a & 1 & -a \\
a & a^{2}\left(\frac{2}{3}-\gamma\right) & -a & a^{2}\left(\frac{4}{3}+\gamma\right)
\end{array}\right] .
$$

The terms of the stiffness matrices with the parameter $\gamma$ are related to the bending matrix $\mathbf{K}_{b}$. The other terms are related to transverse shear matrix $\mathbf{K}_{s}$.

\subsection{4-NODED RECTANGULAR PLATE BENDING FINITE ELEMENT WITH LINEAR SHAPE FUNCTIONS} AND EXACT INTEGRATION

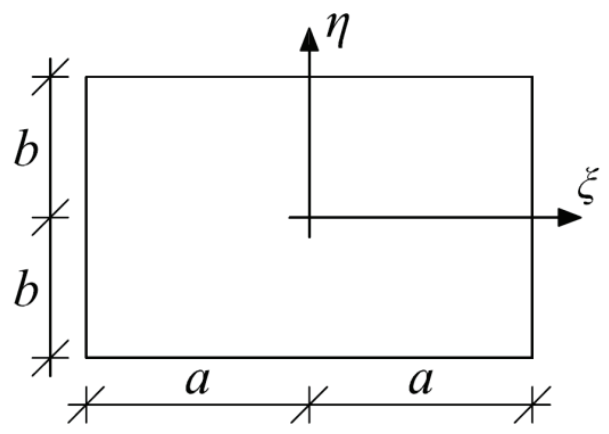

Fig. 1. 4-node rectangular plate bending element.

The subject under consideration is a rectangular moderately thick plate bending element (see Figure 1) on the dimensions $2 a \times 2 b$ and thickness $h$. Let us introduce a non-dimensional co-ordinate system $\xi=\frac{x}{a}, \quad \xi \in\langle-1,1\rangle, \quad \eta=\frac{y}{b}, \quad \eta \in\langle-1,1\rangle$. The nodal parameter vector with corner nodes is natural $\mathbf{q}=\left\{\mathbf{q}_{1}, \mathbf{q}_{2}, \mathbf{q}_{3}, \mathbf{q}_{4}\right\}$, where $\mathbf{q}_{i}=\left\{w_{i}, \phi_{x i}, \phi_{y i}\right\}$. Standard finite element procedure with bi-linear shape functions is introduced 


$$
\begin{gathered}
\mathbf{N}=\left[\mathbf{N}_{1}, \mathbf{N}_{2}, \mathbf{N}_{3}, \mathbf{N}_{4}\right], \quad \mathbf{N}_{i}=\left[\begin{array}{ccc}
N_{i} & 0 & 0 \\
0 & N_{i} & 0 \\
0 & 0 & N_{i}
\end{array}\right], \quad N_{i}=\frac{1}{4}\left(1+\xi_{i} \xi\right)\left(1+\eta_{i} \eta\right), \\
\xi_{1}=-1, \quad \xi_{2}=1, \quad \xi_{3}=1, \quad \xi_{4}=-1, \eta_{1}=-1, \quad \eta_{2}=-1, \quad \eta_{3}=1, \eta_{4}=1 .
\end{gathered}
$$

The element stiffness matrix can be expressed as follows:

$$
\mathbf{K}=\left[\begin{array}{llll}
\mathbf{K}_{11} & \mathbf{K}_{12} & \mathbf{K}_{13} & \mathbf{K}_{14} \\
\mathbf{K}_{21} & \mathbf{K}_{22} & \mathbf{K}_{23} & \mathbf{K}_{24} \\
\mathbf{K}_{31} & \mathbf{K}_{32} & \mathbf{K}_{33} & \mathbf{K}_{34} \\
\mathbf{K}_{41} & \mathbf{K}_{42} & \mathbf{K}_{43} & \mathbf{K}_{44}
\end{array}\right], \quad \mathbf{K}_{i j}=\left[k_{i j}^{k l}\right], \quad i, j=1,2,3,4, k, l=1,2,3,
$$

$$
k_{i j}^{11}=H \frac{\xi_{i} \xi_{j}}{16} \frac{b}{a} 2\left(2+\frac{2}{3} \eta_{i} \eta_{j}\right)+H \frac{\eta_{i} \eta_{j}}{16} \frac{a}{b} 2\left(2+\frac{2}{3} \xi_{i} \xi_{j}\right),
$$$$
k_{i j}^{12}=H \frac{\xi_{i} b}{16} 2\left(2+\frac{2}{3} \eta_{i} \eta_{i}\right), \quad k_{i j}^{13}=H \frac{\eta_{i} a}{16} 2\left(2+\frac{2}{3} \xi_{i} \xi_{j}\right), \quad k_{i j}^{21}=H \frac{\xi_{j} b}{16} 2\left(2+\frac{2}{3} \eta_{i} \eta_{j}\right),
$$$$
k_{i j}^{22}=D \frac{\xi_{i} \xi_{j}}{16} \frac{a}{b} 2\left(2+\frac{2}{3} \eta_{i} \eta_{j}\right)+D \frac{\eta_{i} \eta_{j}}{16} \frac{1-v}{2} \frac{b}{a} 2\left(2+\frac{2}{3} \xi_{i} \xi_{j}\right)+H \frac{a b}{16}\left(2+\frac{2}{3} \xi_{i} \xi_{j}\right)\left(2+\frac{2}{3} \eta_{i} \eta_{j}\right),
$$$$
k_{i j}^{23}=v D \frac{\xi_{i} \eta_{j}}{4}+\frac{1-v}{2} D \frac{\xi_{j} \eta_{i}}{4}, \quad k_{i j}^{31}=H \frac{\eta_{j} a}{16} 4\left(2+\frac{2}{3} \xi_{i} \xi_{j}\right), \quad k_{i j}^{32}=v D \frac{\xi_{j} \eta_{i}}{4}+\frac{1-v}{2} D \frac{\xi_{i} \eta_{j}}{4},
$$

The terms with coefficient $D$ are related to the bending part of the stiffness matrix, as well as the terms with the coefficient $H$ belongs to the shear part. One can observe the locking phenomena in the above finite element (see Gilewski [8]). 


\subsection{Triangular plate bending element DSG (Discrete Shear Gap)}

Let us consider the triangular moderately thick plate bending finite element after Bletzinger and Bischoff [1,2]. Element geometry in orthogonal co-ordinate system $x, y$ is described in Figure 2. We have the natural vector of nodal displacements $\mathbf{q}=\left\{\mathbf{q}_{1}, \mathbf{q}_{2}, \mathbf{q}_{3}\right\}$, where $\mathbf{q}_{i}=\left\{w_{i}, \phi_{x i}, \phi_{y i}\right\}$.

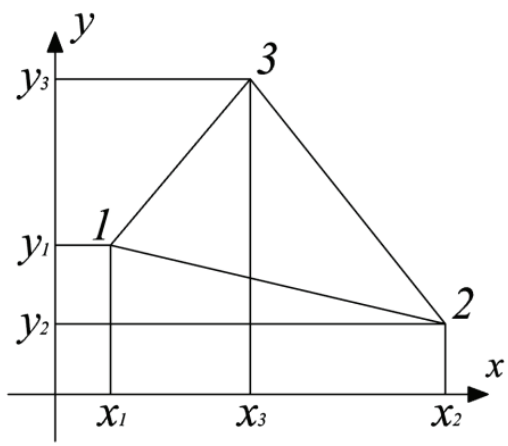

Fig. 2. Triangular plate bending element DSG.

The interested reader is recalled to the reference (Bischoff and Bletzinger [2], Bletzinger, Bishoff and Ramm [3]) for detailed description of the element. The expressions necessary to calculate the stiffness matrix are the following:

$$
\begin{aligned}
& \Delta=\frac{1}{2}\left|\begin{array}{lll}
1 & x_{1} & y_{1} \\
1 & x_{2} & y_{2} \\
1 & x_{3} & y_{3}
\end{array}\right|, \quad \begin{array}{l}
a_{1}=x_{2} y_{3}-x_{3} y_{2}, b_{1}=y_{2}-y_{3}, c_{1}=x_{3}-x_{2}, \\
a_{2}=x_{3} y_{1}-x_{1} y_{3}, b_{2}=y_{3}-y_{1}, c_{2}=x_{1}-x_{3}, \\
a_{3}=x_{1} y_{2}-x_{2} y_{1}, b_{3}=y_{1}-y_{2}, c_{3}=x_{2}-x_{1},
\end{array} \\
& \mathbf{B}_{i}=\frac{1}{2 \Delta}\left[\begin{array}{ccccccccc}
0 & b_{1} & 0 & 0 & b_{2} & 0 & 0 & b_{3} & 0 \\
0 & 0 & c_{1} & 0 & 0 & c_{2} & 0 & 0 & c_{3} \\
0 & c_{1} & b_{1} & 0 & c_{2} & b_{2} & 0 & c_{2} & b_{2} \\
b_{1} & \Delta & 0 & b_{2} & b_{2} c_{3} / 2 & -b_{2} c_{3} / 2 & b_{3} & -b_{3} c_{2} / 2 & -b_{2} b_{3} / 2 \\
c_{1} & 0 & \Delta & c_{2} & c_{2} c_{3} / 2 & -b_{2} c_{2} / 2 & c_{3} & -c_{2} c_{3} / 2 & -b_{2} c_{3} / 2
\end{array}\right] .
\end{aligned}
$$

The element stiffness matrix of the DSG triangular element with 9 d.o.f. has the form 


$$
\mathbf{K}=\left[\mathbf{K}_{i k}\right], \quad \mathbf{K}_{i k}=\iint_{\Omega} \mathbf{B}_{i}^{T} \mathbf{E} \mathbf{B}_{k} d \Omega=\mathbf{B}_{i}^{T} \mathbf{E} \mathbf{B}_{k} \Delta .
$$

The further calculation for the proposed element are to be done for concrete co-ordinates, parameterized by the dimension $a$. Several positions of the triangular element are considered. As it was presented in (Gilewski [8]) the shear locking is observed in the DSG finite element.

\section{ENERGY VERIFICATION OF THE MULTIPLIERS USED IN THE FORMULATION}

The usual method of selection the energy multipliers leads to the number of examples and can be called the "method of tests and mistakes". In the present chapter it is proposed to check easily if and for what value the proposed multipliers can satisfy the consistency condition and can eliminate the terms of parasitic strain energy.

\subsection{2-NODED BEAM ELEMENT}

One can propose to analyze the procedure of verification and selection the stabilization coefficients on the example of 2-noded moderately thick beam finite element with linear shape functions and exact integration.

Step 1 - Definition of the element stiffness matrix.

$$
\mathbf{K}^{2 W-F U L L}=\frac{E J}{2 a^{3}}\left[\begin{array}{cccc}
0 & 0 & 0 & 0 \\
0 & 1 & 0 & -1 \\
0 & 0 & 0 & 0 \\
0 & -1 & 0 & 1
\end{array}\right]+\frac{H}{2 a}\left[\begin{array}{cccc}
1 & a & -1 & a \\
a & \frac{4}{3} a^{2} & -a & \frac{2}{3} a^{2} \\
-1 & -a & 1 & -a \\
a & \frac{2}{3} a^{2} & -a & \frac{4}{3} a^{2}
\end{array}\right]=\mathbf{K}_{b}+\mathbf{K}_{s} .
$$

Step 2 - Identification of the parasitic strain energy terms.

The density of strain energy of the Timoshenko beam in differential form should be as follows

$$
\widetilde{E}_{s}=\frac{1}{2}\left[E J\left(\frac{d \phi}{d x}\right)^{2}+H\left(\frac{d w}{d x}-\phi\right)^{2}\right]
$$

On the other hand the same density should be expressed as a quadratic form regarding the vector $\mathbf{q}$, with the element stiffness matrix as a kernel, divided by the element length 


$$
\widetilde{E}_{s}^{E S}=\frac{1}{2 a} \frac{1}{2} \mathbf{q}^{\mathbf{T}} \mathbf{K q}
$$

The nodal displacements can be expressed by the average displacements and their derivatives with the use of Taylor power series expansion

$$
\begin{aligned}
& w_{1}=w(x)-\frac{\Delta w}{\Delta x}(x) a+\frac{1}{2} \frac{\Delta^{2} w}{(\Delta x)^{2}}(x) a^{2}+\ldots, \quad w_{2}=w(x)+\frac{\Delta w}{\Delta x}(x) a+\frac{1}{2} \frac{\Delta^{2} w}{(\Delta x)^{2}}(x) a^{2}+\ldots \\
& \phi_{1}=\phi(x)-\frac{\Delta \phi}{\Delta x}(x) a+\frac{1}{2} \frac{\Delta^{2} \phi}{(\Delta x)^{2}}(x) a^{2}+\ldots, \quad \phi_{2}=\phi(x)+\frac{\Delta \phi}{\Delta x}(x) a+\frac{1}{2} \frac{\Delta^{2} \phi}{(\Delta x)^{2}}(x) a^{2}+\ldots
\end{aligned}
$$

When we put Eqs. (4.4)-(4.5) to Eq. (4.3) and collect the terms in order to $a$ we can obtain

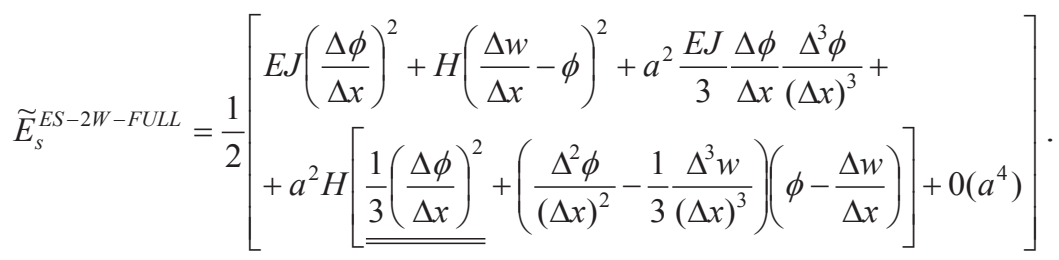

In the limit case, when the element length tends to be zero, we have

$$
\lim _{a \rightarrow 0} \widetilde{E}_{s}^{E S}=\widetilde{E}_{s}
$$

It means that the consistency condition is satisfied. One can observe the doubly underlined parasitic term, responsible for locking of the element. It is better seen if we rewrite the Eq. (4.6) to the following form

$$
\widetilde{E}_{s}^{E S-2 W-F U L L}=\frac{1}{2}\left[\begin{array}{l}
E J\left(1+\frac{1}{\underline{3 \gamma}}\right)\left(\frac{\Delta \phi}{\Delta x}\right)^{2}+k G A\left(\frac{\Delta w}{\Delta x}-\phi\right)^{2}+ \\
+a^{2} H\left[\left(\frac{\Delta^{2} \phi}{(\Delta x)^{2}}-\frac{1}{3} \frac{\Delta^{3} w}{(\Delta x)^{3}}\right)\left(\phi-\frac{\Delta w}{\Delta x}\right)\right]+0\left(a^{4}\right)
\end{array}\right] .
$$


The bending strain energy strongly depends on the parameter $1 / \gamma$ (and in consequence on $\left(\frac{a}{h}\right)^{2}$ ) which is big for thin beams. This energy is called "parasitic" and is large for thin finite elements.

Step 3 - Stabilization.

$\underline{\text { Variant } 1}-\alpha=c \gamma, \beta=0$.

Following the expansion described above the modified strain energy density is in the form

$$
\widetilde{E}_{s}^{E S-2 W-F U L L}=\frac{1}{2}\left[\begin{array}{l}
E J\left(\frac{\Delta \phi}{\Delta x}\right)^{2}+\frac{c E J}{a^{2}}\left(\frac{\Delta w}{\Delta x}-\phi\right)^{2}+a^{2} \frac{E J}{3} \frac{\Delta \phi}{\Delta x} \frac{\Delta^{3} \phi}{(\Delta x)^{3}}+ \\
\left.+c E J\left[\frac{1}{3}\left(\frac{\Delta \phi}{\Delta x}\right)^{2}+\left(\frac{\Delta^{2} \phi}{(\Delta x)^{2}}-\frac{1}{3} \frac{\Delta^{3} w}{(\Delta x)^{3}}\right)\left(\phi-\frac{\Delta w}{\Delta x}\right)\right]+0\left(a^{4}\right)\right]
\end{array}\right] .
$$

It does not satisfy the consistency condition for any numerical value of $c$. This method of stabilization is incorrect from the point of view of the consistency condition.

$\underline{\text { Variant } 2}-\alpha=1-\frac{c}{\gamma}, \beta=0$.

Strain energy density is the following

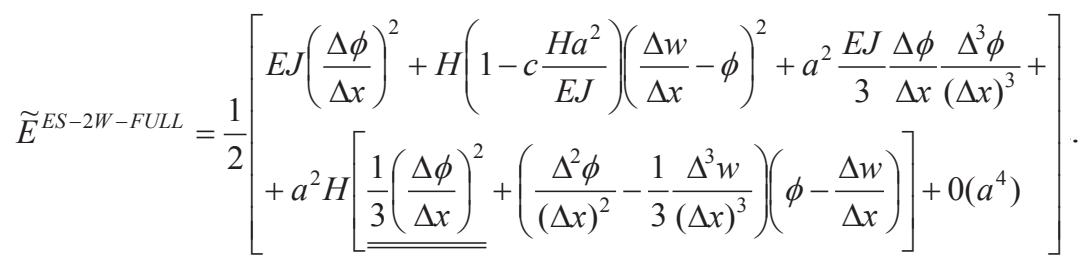

The consistency condition is satisfied because $\lim _{a \rightarrow 0} \widetilde{E}_{s}^{E S}=\widetilde{E}_{s}$. It is possibly to modify the value of the transverse shear strain energy, but the parasitic term (doubly underlined) does not disappear for any $c$. It means that this method of stabilization is formally correct (from the point of view of the consistency condition) but does not allow to cancel the parasitic strains. 
$\underline{\text { Variant } 3}-\alpha=0, \beta=1-\frac{d}{\gamma}$.

Strain energy density is the following

$$
\widetilde{E}_{s}^{E S-2 W-F U L L}=\frac{1}{2}\left[\begin{array}{l}
E J\left(\frac{\Delta \phi}{\Delta x}\right)^{2}+H\left(\frac{\Delta w}{\Delta x}-\phi\right)^{2}+a^{2} \frac{E J}{3} \frac{\Delta \phi}{\Delta x} \frac{\Delta^{3} \phi}{(\Delta x)^{3}}+ \\
\left.+a^{2} H\left[\underline{\underline{\left(\frac{1}{3}-d\right)}\left(\frac{\Delta \phi}{\Delta x}\right)^{2}}+\left(\frac{\Delta^{2} \phi}{(\Delta x)^{2}}-\frac{1}{3} \frac{\Delta^{3} w}{(\Delta x)^{3}}\right)\left(\phi-\frac{\Delta w}{\Delta x}\right)\right]+0\left(a^{4}\right)\right]
\end{array}\right] .
$$

In the limit case $\lim _{a \rightarrow 0} \widetilde{E}_{s}^{E S}=\widetilde{E}_{s}$, so the consistency condition is satisfied. For $d=1 / 3$ the parasitic strain energy term disappears. The stabilized stiffness matrix is the following

$$
\mathbf{K}^{2 W-F U L L-S T A B}=\frac{H}{2 a}\left[\begin{array}{cccc}
1 & a & -1 & a \\
a & a^{2}(1+\gamma) & -a & a^{2}(1-\gamma) \\
-1 & -a & 1 & -a \\
a & a^{2}(1-\gamma) & -a & a^{2}(1+\gamma)
\end{array}\right] .
$$

It is identical to the stiffness matrix received for 2-noded finite element, linear shape functions and with the use of reduced integration (see Gilewski [8]). The spectral analysis of this matrix gives the information thet there are two zero energy modes and the third eigenvalue tends to be zero when the parameter $\gamma \rightarrow 0$. It means that the ellipticity condition can be not satisfied for very thin elements and the stiffness matrix can be over singular. The above example is a proof that the proposed selection of the stabilization of the strain energy allows to elliminate the shear locking and receive satisfied results for thick as well as thin beams.

\subsection{4-NODED PLATE ELEMENT}

Strain energy density of the finite element can be expressed as a quadratic form of the nodal displacements

$$
E_{s}^{E S}=\frac{1}{4 a b} \frac{1}{2} \mathbf{q}^{T} \mathbf{K q}
$$


Each nodal displacement $\mathbf{q}=\left[w_{1}, \phi_{x 1}, \phi_{y 1}, \ldots\right]$ can be expressed by the average displacement and its derivatives with the use of Taylor power series expansion

$$
\begin{aligned}
& f(x+\Delta x, y+\Delta y)=f(x, y)+\frac{\Delta f}{\Delta x}(x, y) \Delta x+\frac{\Delta f}{\Delta y}(x, y) \Delta y+\frac{1}{2} \frac{\Delta^{2} f}{(\Delta x)^{2}}(x, y)(\Delta x)^{2}+ \\
& +\frac{1}{2} \frac{\Delta^{2} f}{(\Delta y)^{2}}(x, y)(\Delta y)^{2}+\frac{\Delta^{2} f}{\Delta x \Delta y}(x, y) \Delta x \Delta y+\frac{1}{6} \frac{\Delta^{3} f}{(\Delta x)^{3}}(x, y)(\Delta x)^{3}+ \\
& +\frac{1}{6} \frac{\Delta^{3} f}{(\Delta x)(\Delta y)^{2}}(x, y)(\Delta x)(\Delta y)^{2}+\frac{1}{6} \frac{\Delta^{3} f}{(\Delta y)(\Delta x)^{2}}(x, y)(\Delta y)(\Delta x)^{2}+\frac{1}{6} \frac{\Delta^{3} f}{(\Delta y)^{3}}(x, y)(\Delta y)^{3}+\ldots .
\end{aligned}
$$

For nodal values we have:

$$
\begin{aligned}
& f_{1}=f(x-a, y-b), f_{2}=f(x+a, y-b), \\
& f_{3}=f(x+a, y+b), f_{4}=f(x-a, y+b),
\end{aligned}
$$

where $f=w, \phi_{x}, \phi_{y}$ and $f_{i}=w_{i}, \phi_{x i}, \phi_{y i}$.

The above expansions are to be put to the quadratic form in Eq. (4.13). Without loss of generality one can consider the square element $a=b$. After collection the terms in order to $a$ the strain energy density will be expressed in the form

$$
\widetilde{E}_{s}^{E S}=L_{11}\left(w, \phi_{x}, \phi_{y} ; \mathbf{E}\right)+a L_{12}\left(w, \phi_{x}, \phi_{y} ; \mathbf{E}\right)+a^{2} L_{13}\left(w, \phi_{x}, \phi_{y} ; \mathbf{E}\right)+\ldots,
$$

where $L_{i j},(i=1, \ldots, 4, j=1,2, \ldots)$ are difference operators.

The analysis of the element without stabilization gives the following results

$$
L_{11}=\frac{1}{2}\left[\begin{array}{l}
D\left(\frac{\Delta \phi_{x}}{\Delta x}\right)^{2}+D \frac{1-v}{2}\left(\frac{\Delta \phi_{x}}{\Delta y}\right)^{2}+D\left(\frac{\Delta \phi_{y}}{\Delta y}\right)^{2}+D \frac{1-v}{2}\left(\frac{\Delta \phi_{y}}{\Delta x}\right)^{2}+ \\
+2 v D \frac{\Delta \phi_{x}}{\Delta x} \frac{\Delta \phi_{y}}{\Delta y}+(1-v) D \frac{\Delta \phi_{y}}{\Delta x} \frac{\Delta \phi_{x}}{\Delta y}+H\left[\left(\phi_{x}-\frac{\Delta w}{\Delta x}\right)^{2}+\left(\phi_{y}-\frac{\Delta w}{\Delta y}\right)^{2}\right]
\end{array}\right],
$$

$$
L_{12}=0
$$




$$
L_{13}=\frac{1}{2}\left[\begin{array}{l}
H \phi_{x}\left(\frac{\Delta^{2} \phi_{x}}{\Delta x^{2}}+\frac{\Delta^{2} \phi_{x}}{\Delta y^{2}}\right)+H \phi_{x}\left(\frac{\Delta^{2} \phi_{y}}{\Delta x^{2}}+\frac{\Delta^{2} \phi_{y}}{\Delta y^{2}}\right)+H \frac{\Delta w}{\Delta x}\left(\frac{\Delta^{2} \phi_{x}}{\Delta x^{2}}+\frac{\Delta^{2} \phi_{x}}{\Delta y^{2}}\right)+ \\
+H \frac{\Delta w}{\Delta y}\left(\frac{\Delta^{2} \phi_{y}}{\Delta x^{2}}+\frac{\Delta^{2} \phi_{y}}{\Delta y^{2}}\right)+\frac{H}{3}\left(\frac{\Delta \phi_{x}}{\Delta x}\right)^{2}+\frac{H}{3\left(\frac{\Delta \phi_{x}}{\Delta y}\right)^{2}+\frac{H}{3}\left(\frac{\Delta \phi_{y}}{\Delta y}\right)^{2}}+\underline{\underline{\underline{\underline{\Delta}}}} \\
+\frac{\frac{H}{3}\left(\frac{\Delta \phi_{y}}{\Delta x}\right)^{2}+\frac{2 H}{3}\left(\frac{\Delta \phi_{x}}{\Delta y}\right)\left(\frac{\Delta^{2} w}{\Delta x \Delta y}\right)+\frac{2 H}{3}\left(\frac{\Delta \phi_{y}}{\Delta x}\right)\left(\frac{\Delta^{2} w}{\Delta x \Delta y}\right)+}{\frac{2 H}{3}\left(\frac{\Delta^{2} w}{\Delta x \Delta y}\right)^{2}+\frac{D(3-v)}{6}\left[\left(\frac{\Delta^{2} \phi_{x}}{\Delta x \Delta y}\right)+\left(\frac{\Delta^{2} \phi_{y}}{\Delta x \Delta y}\right)\right]}
\end{array}\right] .
$$

The comparison of the difference operator $L_{11}$ (Eq. 4.17) with the differential form (Eq. 3.6) shows that the element satisfies the consistency condition. The parasitic terms in the operator $L_{13}$ are doubly underlined.

The use of stabilization coefficient in the form $\beta=1-\frac{d}{\gamma}+\ldots$ does not change the form of difference operators $L_{11}$ and $L_{12}$, what means that the stabilized element remains consistent. The $L_{13}$ difference operator with the use of the stabilized matrix is in the form

$$
\begin{aligned}
& {\left[H \phi_{x}\left(\frac{\Delta^{2} \phi_{x}}{\Delta x^{2}}+\frac{\Delta^{2} \phi_{x}}{\Delta y^{2}}\right)+H \phi_{x}\left(\frac{\Delta^{2} \phi_{y}}{\Delta x^{2}}+\frac{\Delta^{2} \phi_{y}}{\Delta y^{2}}\right)+H \frac{\Delta w}{\Delta x}\left(\frac{\Delta^{2} \phi_{x}}{\Delta x^{2}}+\frac{\Delta^{2} \phi_{x}}{\Delta y^{2}}\right)+\right.} \\
& +H \frac{\Delta w}{\Delta y}\left(\frac{\Delta^{2} \phi_{y}}{\Delta x^{2}}+\frac{\Delta^{2} \phi_{y}}{\Delta y^{2}}\right)+\underline{(1-3 d) \frac{H}{3}\left(\frac{\Delta \phi_{x}}{\Delta x}\right)^{2}}+\underline{\underline{\left(1-3 d \frac{1-v}{2}\right) \frac{H}{3}\left(\frac{\Delta \phi_{x}}{\Delta y}\right)^{2}}}+
\end{aligned}
$$

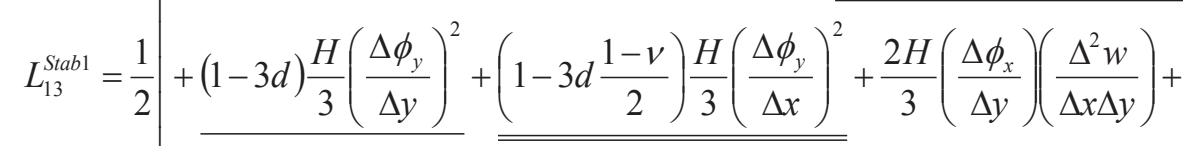

$$
\begin{aligned}
& +\frac{2 H}{3}\left(\frac{\Delta \phi_{y}}{\Delta x}\right)\left(\frac{\Delta^{2} w}{\Delta x \Delta y}\right)+\frac{2 H}{3}\left(\frac{\Delta^{2} w}{\Delta x \Delta y}\right)^{2}+\frac{D(3-v)}{6}\left[\left(\frac{\Delta^{2} \phi_{x}}{\Delta x \Delta y}\right)+\left(\frac{\Delta^{2} \phi_{y}}{\Delta x \Delta y}\right)\right]+ \\
& \underline{\underline{-d H(1-v) \frac{\Delta \phi_{x}}{\Delta y} \frac{\Delta \phi_{y}}{\Delta x}}} \overline{\underline{2 d H v \frac{\Delta \phi_{x}}{\Delta x} \frac{\Delta \phi_{y}}{\Delta y}}}
\end{aligned}
$$


The single underlined parasitic term can be eliminated by taking $d=1 / 3$ or the double underlined term by taking $d=\frac{2}{3(1-v)}$. Two other, new parasitic terms doubly under- and overlined cannot be eliminated. It is well seen that this version of stabilization coefficient cannot fully eliminate the strain locking with the use on one parameter $d$ and two additional parasitic terms appeared in comparison with the not stabilized matrix.

Let us propose the way for introducing the stabilization with the use of multi-coefficients. Assume the elasticity matrix in the following form

$$
\mathbf{E}=\left[\begin{array}{ccccc}
D \beta_{1} & v D \beta_{2} & 0 & 0 & 0 \\
v D \beta_{2} & D \beta_{3} & 0 & 0 & 0 \\
0 & 0 & \frac{1-v}{2} D \beta_{4} & 0 & 0 \\
0 & 0 & 0 & H & 0 \\
0 & 0 & 0 & 0 & H
\end{array}\right], \quad \beta_{i}=1-\frac{d_{i}}{\gamma}, \quad i=1,2,3,4
$$

In this formulation we have four different coefficients that can be used to eliminate the parasitic terms.

If we use the stabilization in the form Eq. (4.21) the difference operators $L_{11}$ and $L_{12}$ remain unchanged. The third operator is in the form

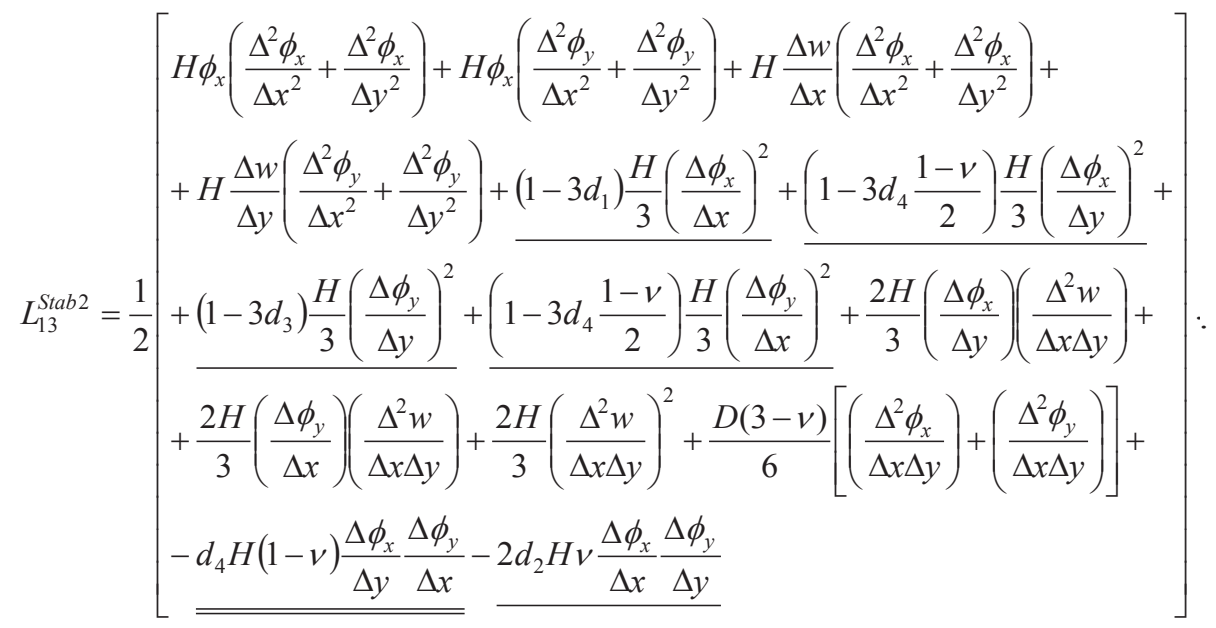

Putting $d_{1}=1 / 3, d_{2}=0, d_{3}=1 / 3, d_{4}=\frac{2}{3(1-v)}$ we can eliminate the parasitic terms which are single underlined. Unfortunately it was not possible to eliminate the doubly underlined term. 


\subsection{Triangular DSG plate element}

The DSG triangular plate bending element has the parasitic energy terms that depends on the element geometry and the sequence of numbering the nodal points (see Gilewski [8]). The method of stabilization described above always leads to satisfy the consistency condition, what means that the $L_{11}$ difference operator is in agreement with the differential one. For triangular elements the operators $L_{12} \neq 0$, but there are no parasitic energy terms there. The form of $L_{13}$ difference operator depends on the element geometry. Three different positions of the element nodes are analyzed below (see Figure 3). The element geometries are parameterized with the use of , $a$ ". The first element is irregular. The second and third are regular and typical for not concentrated triangular meshes.

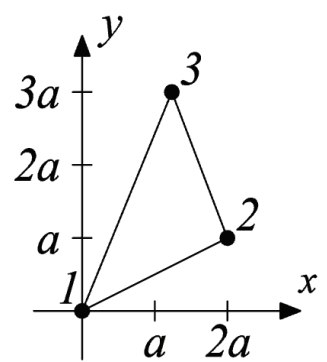

Position 1

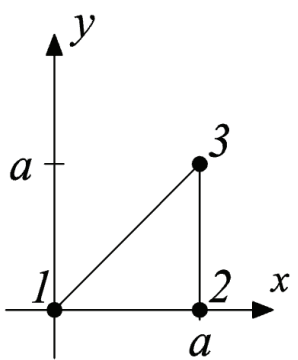

Position 2

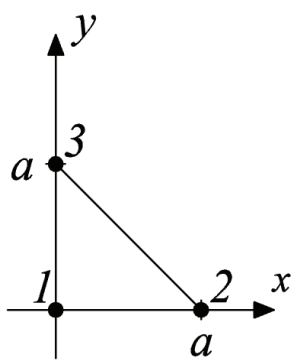

Position 3

Fig. 3. Positions of DSG triangular elements.

The form of parasitic energy terms in the difference operators $L_{13}$, when the extended elasticity matrix (4.21) is applied, is the following:

\section{Position 1}

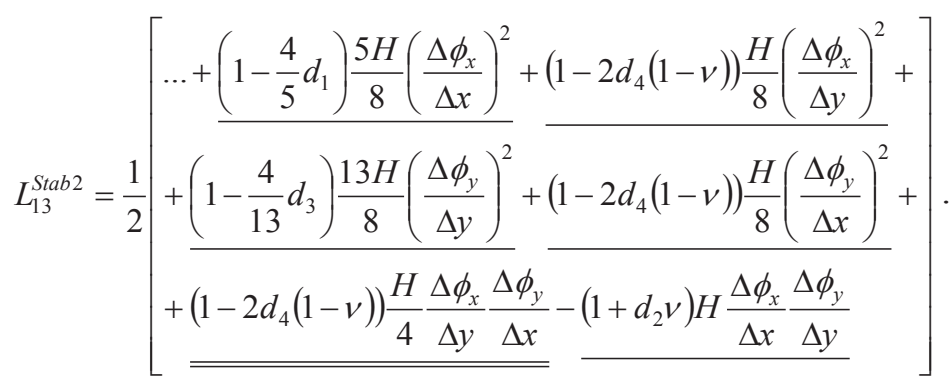


Putting $d_{1}=\frac{5}{4}, d_{2}=-\frac{1}{v}, d_{3}=\frac{13}{4}, d_{4}=\frac{1}{2(1-v)}$ one can eliminate all parasitic energy terms and have the element totally free from locking.

\section{Position 2}

$$
L_{13}^{\text {Stab2 }}=\frac{1}{2}\left[\begin{array}{c}
\frac{\left(1-4 d_{1}\right) \frac{H}{8}\left(\frac{\Delta \phi_{x}}{\Delta x}\right)^{2}+\left(1-2 d_{4}(1-v)\right) \frac{H}{8}\left(\frac{\Delta \phi_{x}}{\Delta y}\right)^{2}}{+}+ \\
+\frac{\left(1-4 d_{3}\right) \frac{H}{8}\left(\frac{\Delta \phi_{y}}{\Delta y}\right)^{2}+\left(1-2 d_{4}(1-v)\right) \frac{H}{8}\left(\frac{\Delta \phi_{y}}{\Delta x}\right)^{2}}{+\left(1-2 d_{4}(1-v)\right) \frac{H}{4} \frac{\Delta \phi_{x}}{\Delta y} \frac{\Delta \phi_{y}}{\Delta x}-d_{2} H v \frac{\Delta \phi_{x}}{\Delta x} \frac{\Delta \phi_{y}}{\Delta y}}
\end{array}\right] .
$$

The parameters to eliminate locking effects are

$$
d_{1}=\frac{1}{4}, d_{2}=0, d_{3}=\frac{1}{4}, d_{4}=\frac{1}{2(1-v)} .
$$

\section{Position 3}

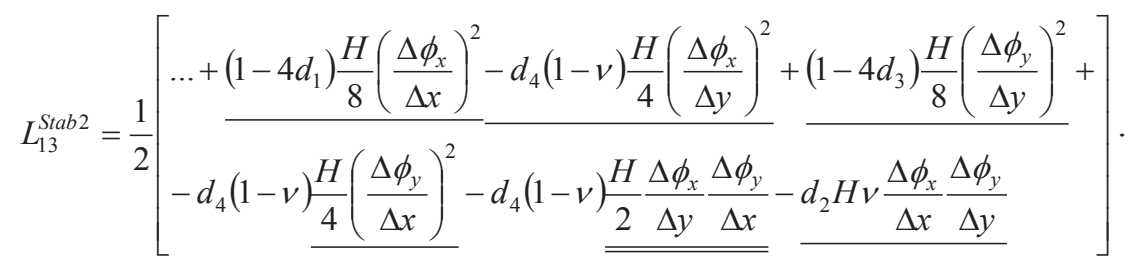

The parameters to eliminate locking effects are

$$
d_{1}=\frac{1}{4}, d_{2}=0, d_{3}=\frac{1}{4}, d_{4}=0
$$

\section{Conclusions}

In the present paper the possibility of the use of energy multipliers for stabilization the parasitic strain energy and elimination of locking for moderately thick beam and plate elements are discussed. 
Three ways of introducing the correction coefficients were presented and discussed on the example of Timoshenko beam 2-node finite element. The first way, known in the literature, does not satisfy the consistency condition and is incorrect. The second, also known in the literature, is correct from the point of view of the consistency condition, but does not allow to eliminate the parasitic strain energy terms. The third way, proposed in this paper, allow to eliminate parasitic strains and locking and is consistent. The proposed way leads to the stiffness matrix identical to the method of reduced integration.

A similar way applied to the plate element wasn't fully successful. A new 4-parameter idea of construction of the stabilized matrix was proposed. For the 4-noded rectangular element with linear shape functions it was possible to eliminate 5 of 6 parasitic strain energy terms. For triangular DSG element it was possible to fully eliminate parasitic terms. The numerical values of the coefficients depend on the element geometry.

The similar procedure can be used for other beam, plate and shell finite elements.

\section{REFERENCES}

1. M. Bischoff, K.U. Bletzinger K.U., Improving stability and accuracy of Reissner-Mindlin plate finite elements via algebraic subgird scale stabilization, Comp. Meth. Appl. Mech. Engrg., 193, 1517-1528, 2004

2. M. Bischoff, K.U. Bletzinger, Stabilized DSG plate and shell elements", In: "Trends in Computational Structural Mechanics, W. A. Wall, at al., eds., CIMNE, Barcelona, 253-263, 2001

3. K.U. Bletzinger, M. Bischoff, E. Ramm, A unified approach for shear-locking free triangular and rectangular shell finite elements, Computers \& Structures, 75, 321-334, 2000

4. N. Carpenter, T. Belytschko, H. Stolarski, Locking and shear scaling factors in C-0 bending elements, Computers \& Structures, 22, 39-52, 1986

5. H.R. Dhananjaya, P.C. Pandey, J. Nagabhushanam, I. Zainah, New nine-node Lagrangian quadrilateral plate element based on Mindlin-Reissner theory using IFM, An International Journal of Structural Engineering and Mechanics, 41, 205-229, 2012

6. I. FrIED I, Shear in C-0 and C-1 bending elements, Int. Journ. Solids. Struct., 11, 449-460, 1973

7. W. Gilewski, Correctness of plate bending element with physical shape functions, Finite Element News, 3, 29-34, 1993

8. W. Gilewski, On the Criteria for Evaluation of Finite Elements: From Timoshenko Beam to HenckyBolle Plate [in Polish], Warsaw University of Technology Publishing House, Warsaw, Poland, 2005

9. W. Gilewski, Some extensions of energy difference criterion for finite element evaluation, CMM-2007, Łódź-Spała, 2007

10. W. Gilewski, M. Sitek, The inf-sup condition tests for shell/plate finite elements, Archives of Civil Engineering, LVII, 425-447, 2011

11. A. Iosilevich, K.J. Bathe, F. Brezzi, On evaluation the inf-sup condition for plate bending elements, International Journal for Numerical Methods in Engineering, 40, 3639-3663, 1997

12. M. Lyly, R. Stenberg, T. Vihinen, A stable bilinear element for the Reissner-Mindlin plate model, Comp. Meth. Appl. Mech. Engrg., 110, 343-357, 1993

13. G.A. Монr, Application of penalty functions to a curved isoparametric axisymmetric thick shell element, Computers \& Structures, 15, 685-690, 1982 
14. M. Rezaiee-Pajand, F. Shahabian, F.H. Tavakoli, A new higher-order triangular plate bending element for the analysis of laminated composite and sandwich plates, An International Journal of Structural Engineering and Mechanics, 43, 253-271, 2012

15. A. Tessler, Shear deformable bending elements with penalty relaxation, In: Finite Element Methods for Plate and Shell Structures, Vol. 1. Element Technology, T.J.R. Hughes, E. Hinton, eds., Pineridge Press, Swensea, 266-290, 1986

Received: 15.04 .2014

Revised: 12.09 .2014 